\title{
WITHDRAWN: Resource poor countries ought to focus on early detection and containment of novel corona virus at the point of entry
}

Krishna Prasad Acharya

Animal Quarantine Office (AQO), Department of Livestock Services (DLS), Kathmandu, Nepal

This article has been withdrawn at the request of the author(s) and/ or editor. The Publisher apologizes for any inconvenience this may cause.
The full Elsevier Policy on Article Withdrawal can be found at https://www.elsevier.com/about/our-business/policies/articlewithdrawal

E-mail addresses: krishna.acharya@nepal.gov.np, kriaasedu@gmail.com. 